(1)

CrossMark

\title{
Distinct features of SARS-CoV-2-specific IgA response in COVID-19 patients
}

\author{
To the Editor:
}

In comparison to severe acute respiratory syndrome coronavirus (SARS-CoV), SARS-CoV-2 appears to be more contagious [1], and coronavirus disease 2019 (COVID-19) patients demonstrate varied clinical manifestations distinct from those seen in patients with SARS-CoV and Middle East respiratory syndrome coronavirus infections [2]. Collective results from the clinical and epidemiological observations suggest a distinct viral-host interaction in COVID-19 patients. Profiling of the antibody response during SARS-CoV-2 infection may help improve our understanding of the viral-host interaction and the immunopathological mechanisms of the disease.

Studies on humoral responses to infections have been mainly geared toward the production of high-affinity IgG antibodies that efficiently resolve an infection. It has been well recognised, however, that humoral immune response to infection can be a double-edged sword that either serves as a protective mechanism to resolve the infection or aggravates the tissue injury, e.g. the IgG response causes fatal acute lung injury by skewing the inflammation-resolving response in SARS-CoV [3]. In the case of respiratory infection, while IgM and IgG isotypes have been the primary emphasis in characterising immunity, mucosal and systemic IgA responses that may play a critical role in the disease pathogenesis have received much less attention.

This study was designed to better understand the timing and patterns of humoral immune responses to SARS-CoV-2 in a cohort of COVID-19 patients and evaluate their relationship with the disease course and severity. 37 patients with COVID-19, with a mean \pm SD age of $52.3 \pm 16.3$ years, were enrolled in this study. The enrolled COVID-19 patients consisted of 25 (67.6\%) males and 12 (32.4\%) females. All patients tested positive for viral nucleic acid of SARS-CoV-2 (Real-Time Fluorescent RT-PCR Kit; BGI, Shenzhen, China). According to the "Guidelines for the Diagnosis and Treatment of Novel Coronavirus (2019-nCoV) Infection" published by the National Health Commission of China, the enrolled COVID-19 patients were categorised into two groups: 20 (54.1\%) severe cases and 17 (46.0\%) nonsevere cases [4]. The nonsevere group included patients with mild and moderate symptoms who were also required to be admitted to hospital by the COVID-19 control policy in China. The severe group included severe and critically ill patients. Mild patients did not demonstrate abnormal computed tomography (CT) imaging. Moderate patients had fever and/or classical respiratory symptoms, and typical CT images of viral pneumonia. Severe patients met at least one of following additional conditions: 1) shortness of breath with a respiratory rate $\geqslant 30$ times $\min ^{-1} ; 2$ ) oxygen saturation measured by pulse oximetry (resting state) of $\leqslant 93 \%$; or 3 ) arterial oxygen tension/inspiratory oxygen tension of $\leqslant 300 \mathrm{mmHg}$. Critically ill patients met at least one of the extra following conditions in addition to the COVID-19 diagnosis: 1) respiratory failure that required mechanical ventilation; 2) shock; or 3) multiple organ failure that required transfer to the intensive care unit. All clinical diagnoses were confirmed by a team of trained physicians. All blood samples were collected within a timeframe of 0-8 weeks after admission. A total of 183 serum samples collected during the hospitalisation period from the 37 patients were tested for SARS-CoV-2 spike (S) protein-specific antibodies. The levels of SARS-CoV-2 S protein-specific IgA, IgM and IgG antibodies were assayed using chemiluminescent immunoassay. The S-protein peptide was acquired from the University of Science and Technology of China (Hefei, China).

@ERSpublications

Humoral immune response to SARS-CoV-2 showed an early response of IgA, instead of IgM, in COVID-19 patients. As highlighted by this study, enhanced IgA responses observed in severe COVID19 might confer damaging effects in severe COVID-19. https://bit.ly/3fA7clI

Cite this article as: Yu H-qiong, Sun B-qing, Fang Z-fu, et al. Distinct features of SARS-CoV-2-specific IgA response in COVID-19 patients. Eur Respir J 2020; 56: 2001526 [https://doi.org/10.1183/ 13993003.01526-2020]. 
Our study shows that the first seroconversion day of IgA was 2 days after onset of the initial symptoms, and the first seroconversion day of IgM and IgG was 5 days after onset. The positive rate of antibodies in the 183 samples was $98.9 \%, 93.4 \%$ and $95.1 \%$, for $\operatorname{IgA}$, IgM and IgG, respectively. The seroconversion rate for IgA, IgM or IgG was $100 \% 32$ days after symptom onset. According to the cumulative seroconversion curve, the median conversion time for IgA, IgM and IgG was 13, 14 and 14 days, respectively. The levels of both Ag-specific IgA and IgG were markedly increased $~ 2$ weeks after symptom onset and remained continuously elevated for the following 2 weeks. In contrast, the levels and time-dependent changes of IgM were minimal. The relative levels of IgA and IgG were markedly higher in severe patients compared with nonsevere patients (figure 1). There were significant differences in the relative levels of $\operatorname{IgA}(\mathrm{p}<0.001)$ and $\operatorname{IgG}(\mathrm{p}<0.001)$ between the severe and nonsevere groups. In contrast, no statistically significant changes occurred in the levels of IgM between the severe and nonsevere patients after disease onset. In further subgroup analysis, we found a significant positive association between the level of SARS-CoV-2-specific IgA and the Acute Physiology and Chronic Health Evaluation (APACHE)-II score in critically ill patients with COVID-19 ( $\mathrm{r}=0.72, \mathrm{p}=0.01)$, while the level of SARS-CoV-2-specific IgG and IgM did not show correlations with disease severity.

The present study shows that the levels of specific IgM antibody were significantly lower than those of IgA in both severe and nonsevere patents. This pattern of humoral immune response is different in SARS-CoV infection, in which IgM and IgA show similar chronological profiles in terms of both seroconversion time and antibody titres [5], in line with the knowledge that viremia is common in SARS.

As a mucosal targeted virus, SARS-CoV-2 would be expected to generate secretory IgA (sIgA) and induce strong mucosal immunity. Indeed, mucosal anti-viral immunity has been shown to result in part from IgA-mediated interactions with pathogenic microorganisms to prevent pathogens from adhering to the cell surface [6]. However, recent studies have also found that sIgA is able to induce interleukin (IL)-6, IL-8, monocyte chemoattractant protein-1 and granulocyte-macrophage colony stimulating factor production through normal human lung fibroblasts [7]. It has also been proposed that SIgA may have synergistic effects with IgG in promoting antibody-dependent cellular cytotoxicity (ADCC) [8]. In contrast with mucosal IgA, the role of serum IgA is relatively unexplored. Previous studies have shown that IgA mediates either pro- or anti-inflammatory effects in innate immune cells and have suggested a plausible role of IgA as a driver of
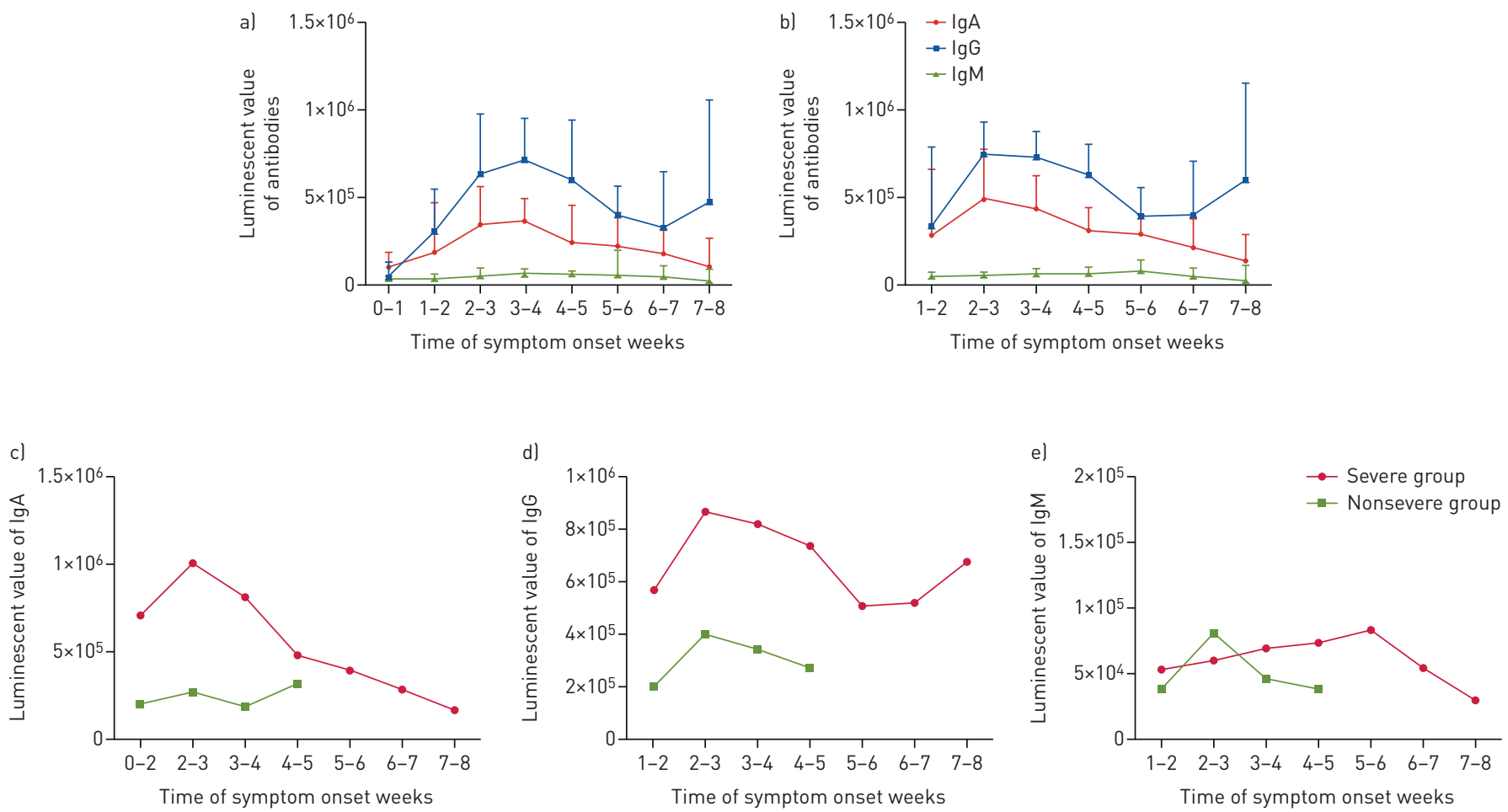

FIGURE 1 Chronological profiles of antibodies in patients with coronavirus disease 2019. Samples collected since illness onset and during weeks $0-1,1-2,2-3,3-4,4-5,5-6,6-7$ and $7-8$ were pooled for analysis. a and b) The medians of the antibody detection values (luminescent values) of samples at the same time-point since onset in al all patients and b) patients with severe illness. c-e) Time-serial changes of the levels of different antibodies in severe and nonsevere groups. The medians of antibody detection values (luminescent values) of samples at the same time-point since onset for c) $\lg A$, d) $\lg G$ and e) $\lg M$. 
autoimmune diseases and as a regulator of immune hyperactivation [9]. Monomeric binding of serum IgA to the $\mathrm{Fc}$ alpha receptor (FcoRI) has been suggested to mediate inhibitory function via the receptor inhibitory signals in a variety of myeloid cells [10]. In contrast, crosslinking of FcoRI by IgA and pathogen is able to transmit activating signals, leading to phagocytosis, respiratory burst, ADCC, increased antigen presentation, degranulation and cytokine release [11]. Cytokines such as transforming growth factor (TGF)- $\beta$ and IL-10 can induce antibody isotype switching [12]. Upregulated IgA production may be the result of increased levels of TGF- $\beta$ and IL-10, which promote antibody switching in SARS-CoV-2 infection.

Considering the roles of mucosal and systemic IgA in COVID-19, inducing IgA production (using lactoferrin to activate canonical TGF- $\beta$ signalling [13] or retinoic acid to enhance lactoferrin-induced IgA responses [14]) has been proposed as a novel therapy for severe COVID-19. However, as highlighted by our study, enhanced IgA responses observed in severe COVID-19 might confer damaging effects in severe COVID-19. As a result, we hypothesise that severe COVID-19 might be at least in part an IgA-mediated disease (related to IgA deposition and vasculitis), which helps to explain common organ injuries in COVID-19, e.g. acute pulmonary embolism, kidney injury, etc. [15]. We acknowledge the limitations of this study, including the fact that there was no measurement of local IgA and the limited number of patients. Future investigative efforts are certainly needed to explore the functional significance of mucosal and systemic IgA in COVID-19 and whether local interventions at the mucosal level of the nasopharynx/ oropharynx could reduce viral load and symptoms.

Hai-qiong $\mathrm{Yu}^{1,9}$, Bao-qing Sun ${ }^{2,9}$, Zhang-fu Fang ${ }^{1,2,3,9}$, Jin-cun $\mathrm{Zhao}^{2,9}$, Xiao-yu Liu ${ }^{1,9}$, Yi-min $\mathrm{Li}^{2,9}$, Xizhuo Sun ${ }^{3,9}$, Hong-feng Liang ${ }^{4,9}$, Bei Zhong ${ }^{5,9}$, Zhi-feng Huang ${ }^{2,9}$, Pei-yan Zheng ${ }^{2}$, Li-feng Tian ${ }^{6}$, Hui-Qi Qu $\oplus^{6}$, De-chen $\mathrm{Liu}^{7}$, Er-yi Wang, Xiao-jun Xiao ${ }^{1}$, Shi-yue $\mathrm{Li}^{2}$, Feng $\mathrm{Ye}^{2}$, Li Guan, Dong-sheng $\mathrm{Hu}^{3}$, Hakon Hakonarson ${ }^{6,8,10}$, Zhi-gang Liu ${ }^{1,10}$ and Nan-shan Zhong ${ }^{2,10}$

${ }^{1}$ State Key Laboratory of Respiratory Disease for Allergy at Shenzhen University, Shenzhen Key Laboratory of Allergy and Immunology, Shenzhen University School of Medicine, Shenzhen, China. ${ }^{2}$ State Key Laboratory of Respiratory Disease, National Clinical Research Center for Respiratory Disease, Guangzhou Institute of Respiratory Health, First Affiliated Hospital of Guangzhou Medical University, Guangzhou, China. ${ }^{3}$ Dept of Respirology and Allergy, Third Affiliated Hospital of Shenzhen University, Shenzhen, China. ${ }^{4}$ Yangjiang People's Hospital, Yangjiang, China. ${ }^{5}$ Dept of Infectious Disease, The Sixth Affiliated Hospital of Guangzhou Medical University, Qingyuan People's Hospital, Qingyuan, China. ${ }^{6}$ Center for Applied Genomics, The Children's Hospital of Philadelphia, Philadelphia, PA, USA. ${ }^{7}$ Dept of Preventive Medicine, Shenzhen University Health Sciences Center, Shenzhen, China. ${ }^{8}$ Divisions of Human Genetics and Pulmonary Medicine, Dept of Pediatrics, The Perelman School of Medicine, University of Pennsylvania, Philadelphia, PA, USA. ${ }^{9}$ These authors contributed equally to this work. ${ }^{10}$ Hakon Hakonarson, Zhi-gang Liu and Nanshan Zhong are joint lead authors.

Correspondence: Nan-shan Zhong, State Key Laboratory of Respiratory Disease, Guangzhou Institute of Respiratory Health, The First Affiliated Hospital of Guangzhou Medical University, 151 Yanjiang Rd., Guangzhou (510120), China. E-mail: nanshan@vip.163.com

Received: 29 April 2020 | Accepted after revision: 5 May 2020

Acknowledgements: We thank all the patients and their families involved in this study, as well as doctors, nurses and civilians that had worked hard to fight against SARS-CoV-2. We would like to express great thankfulness to Professor Shau-Ku Huang (National Health Research Institutes, Miaoli, Taiwan) for his suggestions and critical reading of this manuscript.

Author contributions: Hai-qiong Yu, Bao-qing Sun, Zhang-fu Fang, Jin-cun Zhao, Xiao-yu Liu, Yi-min Li, Xi-zhuo Sun, Zhi-gang Liu and Nan-shan Zhong participated in the study design. Hong-feng Liang, Bei Zhong, Zhi-feng Huang, Pei-yan Zheng, Li-feng Tian, Hui-Qi Qu, De-chen Liu, Er-yi Wang, Xiao-jun Xiao, Shi-yue Li, Feng Ye, Li Guan and Dong-sheng Hu contributed to patient recruitment, data collection, data analysis and literature search. Zhang-fu Fang, Zhi-feng Huang and Pei-yan Zheng conducted the experiments on antibody detection. Hai-qiong Yu, Bao-qing Sun, Zhang-fu Fang, Li-feng Tian, Hui-Qi Qu and Hakon Hakonarson drafted the manuscript. Nan-shan Zhong and Zhi-gang Liu critically reviewed and revised the manuscript. All authors read the manuscript and approved the final version.

Conflict of interest: Hai-qiong Yu has nothing to disclose. Bao-qing Sun has nothing to disclose. Zhang-fu Fang has nothing to disclose. Jin-cun Zhao has nothing to disclose. Xiao-yu Liu has nothing to disclose. Yi-min Li has nothing to disclose. Xi-zhuo Sun has nothing to disclose. Hong-feng Liang has nothing to disclose. Bei Zhong has nothing to disclose. Zhi-feng Huang has nothing to disclose. Pei-yan Zheng has nothing to disclose. Li-feng Tian has nothing to disclose. Hui-Qi Qu has nothing to disclose. De-chen Liu has nothing to disclose. Er-yi Wang has nothing to disclose. Xiao-jun Xiao has nothing to disclose. Shi-yue Li has nothing to disclose. Feng Ye has nothing to disclose. Li Guan has nothing to disclose. Dong-sheng Hu has nothing to disclose. Hakon Hakonarson has nothing to disclose. Zhi-gang Liu has nothing to disclose. Nan-shan Zhong has nothing to disclose.

Support statement: This work was funded by grants from Shenzhen Science and technology plan research project (No. KQTD20170331145453160), Shenzhen Nanshan District Pioneer Group Research Funds (No.LHTD20180007), Zhejiang University special scientific research fund for COVID-19 prevention and control (2020XGZX001,2020XGZX025). The research was designed, conducted, analysed and interpreted by the authors entirely independently of the funding sources. 


\section{References}

1 Sanche S, Lin YT, Xu C, et al. High contagiousness and rapid spread of severe acute respiratory syndrome coronavirus 2. Emerging Infect Dis 2020; 26: 1470-1477.

2 Wang C, Horby PW, Hayden FG, et al. A novel coronavirus outbreak of global health concern. Lancet 2020; 395: 470-473.

3 Liu L, Wei Q, Lin Q, et al. Anti-spike IgG causes severe acute lung injury by skewing macrophage responses during acute SARS-CoV infection. JCI Insight 2019; 4: 123158.

4 Guidelines for the Diagnosis and Treatment of Novel Coronavirus (2019-nCoV) Infection by the National Health Commission (Trial Version 7). http://www.gov.cn/zhengce/zhengceku/2020-03/04/content_5486705.htm Date last accessed: 4 May 2020. Date last updated: 3 March 2020.

5 Hsueh P-R, Huang L-M, Chen P-J, et al. Chronological evolution of IgM, IgA, IgG and neutralisation antibodies after infection with SARS-associated coronavirus. Clin Microbiol Infect 2004; 10: 1062-1066.

6 Corthesy B. Role of secretory IgA in infection and maintenance of homeostasis. Autoimmun Rev 2013; 12: 661-665.

7 Arakawa S, Suzukawa M, Watanabe K, et al. Secretory immunoglobulin A induces human lung fibroblasts to produce inflammatory cytokines and undergo activation. Clin Exp Immunol 2019; 195: 287-301.

8 Shen L, Fanger MW. Secretory IgA antibodies synergize with IgG in promoting ADCC by human polymorphonuclear cells, monocytes, and lymphocytes. Cell Immunol 1981; 59: 75-81.

9 Olas K, Butterweck H, Teschner W, et al. Immunomodulatory properties of human serum immunoglobulin A anti-inflammatory and pro-inflammatory activities in human monocytes and peripheral blood mononuclear cells. Clin Exp Immunol 2005; 140: 478-490.

10 Oortwijn BD, Roos A, van der Boog PJ, et al. Monomeric and polymeric IgA show a similar association with the myeloid FcoRI/CD89. Mol Immunol 2007; 44: 966-973.

11 Leong KW, Ding JL. The unexplored roles of human serum IgA. DNA Cell Biol 2014; 33: 823-829.

12 Dullaers M, Li D, Xue Y, et al. A T cell-dependent mechanism for the induction of human mucosal homing immunoglobulin A-secreting plasmablasts. Immunity 2009; 30: 120-129.

13 Jang YS, Seo GY, Lee JM, et al. Lactoferrin causes IgA and IgG2b isotype switching through betaglycan binding and activation of canonical TGF- $\beta$ signaling. Mucosal Immunol 2015; 8: 906-917.

14 Lee J-M, Jang Y-S, Jin B-R, et al. Retinoic acid enhances lactoferrin-induced IgA responses by increasing betaglycan expression. Cell Mol Immunol 2016; 13: 862-870.

15 Danzi GB, Loffi M, Galeazzi G, et al. Acute pulmonary embolism and COVID-19 pneumonia: a random association? Eur Heart J 2020; 41: 1858. 